\title{
EMPREGO DE ÓXIDOS TIPO PEROVSKITA NAS OXIDAÇÕES DO PROPANO E CO
}

\author{
Paulo Roberto Nagipe da Silva
}

Centro de Ciência e Tecnologia, Universidade Estadual do Norte Fluminense, Av. Alberto Lamego, 2000, 28015-620 Campos dos Goytacazes - RJ

Recebido em 29/11/02; aceito em 8/9/03

\begin{abstract}
EMPLOYMENT OF PEROVSKITE -TYPE OXIDE IN THE PROPANE AND CO OXIDATIONS. Simultaneous oxidation/coprecipitation of an equimolar mixture of $\mathrm{La}(\mathrm{III})$ and $\mathrm{Co}$ (II) nitrates and $\mathrm{La}$ (III) nitrate and $\mathrm{Mn}$ (II) chloride afforded a hydroxide gel, which was converted to $\mathrm{LaCoO}_{3}$ and $\mathrm{LaMnO}_{3}$ on calcination at $600{ }^{\circ} \mathrm{C}$. After calcination, the obtained perovskites have been characterised by X-ray diffraction (XRD), X- ray photoelectron spectroscopy (XPS), thermogravimetric analysis (DTA TGA) and BET specific surface determination. Specific surface areas of perovskites were $12-60 \mathrm{~m}^{2} / \mathrm{g}$. XRD analysis showed that $\mathrm{LaCoO}_{3}$ and $\mathrm{LaMnO}_{3}$ are simple phase perovskite - type oxides. Traces of $\mathrm{LaOCl}$, in addition to the perovskite were detected in the $\mathrm{LaMnO}_{3}$. The catalytic behavior was examined in the propane and $\mathrm{CO}$ oxidation. The $\mathrm{LaCoO}_{3}$ catalyst was more active to $\mathrm{CO}_{2}$ than the $\mathrm{LaMnO}_{3}$ catalyst.
\end{abstract}

Keywords: perovskites; $\mathrm{CO}$ oxidation, propane oxidation.

\section{INTRODUÇÃO}

Os óxidos tipo perovskitas têm a fórmula geral $\mathrm{ABO}_{3 \pm \delta}$, sendo $\mathrm{A}$ um cátion grande tal como os metais alcalinos, os alcalinos terrosos e as terras raras; B um cátion menor, tal como um metal de transição. As perovskitas mais comuns são aquelas em que A é um cátion de um elemento terra rara com estado de oxidação +3 e B um metal de transição no mesmo estado de valência. Esses materiais possuem uma estrutura bem definida, sendo a perovskita ideal do tipo cúbico. Entretanto, essas estruturas aparecem freqüentemente distorcidas, principalmente para as simetrias ortorrômbicas e romboédricas ${ }^{1}$. É possível realizar substituições parciais dos cátions A e B sem alterar a estrutura fundamental da perovskita ${ }^{2}$. Muita atenção tem sido dada às perovskitas à base de lantânio $\left(\mathrm{LaBO}_{3}\right), \mathrm{B}=\mathrm{Co}$ ou $\mathrm{Mn}$, devido à sua alta atividade e estabilidade térmica em reações de oxidação de hidrocarbonetos. No caso do cobalto, as perovskitas correspondentes são denominadas de cobaltitas de lantânio $\left(\mathrm{LaCoO}_{3}\right)$ e no caso do manganês, de manganitas de lantânio $\left(\mathrm{LaMnO}_{3}\right)$. Essas perovskitas chegam a ter atividades comparáveis às dos metais nobres ${ }^{2}$. Entre as perovskitas de lantânio a manganita é a única rica em oxigênio ${ }^{3}$, isto é $\delta>0$. De outro lado, as manganitas podem tornar-se deficientes em oxigênio pela introdução de um outro sítio catiônico $\mathrm{B}$, como é o caso do ferro ${ }^{4}$.

O efeito da substituição parcial dos cátions A, geralmente um metal terra rara, com elementos que apresentam estados de valência diferente de +3 , tem sido freqüentemente estudado ${ }^{5,6}$, enquanto o efeito da substituição de cátions do tipo B, e sua aplicação na combustão catalítica tem despertado menos o interesse dos pesquisadores ${ }^{7}$.

O interesse inicial pelo estudo desses materiais foi motivado por suas propriedades ótica, elétrica e magnética. Comercialmente, as perovskitas de elementos terras raras têm sido muito aplicadas na fabricação de eletrodos ${ }^{8}$. Na catálise, vários trabalhos na área ambiental têm demonstrado a versatilidade das perovskitas contendo Co e Mn como metais de transição. Assim, as perovskitas tem sido aplicadas na redução seletiva de óxido nítrico com propeno ${ }^{9}$, na oxidação de $\mathrm{CO}$ e hidrocarbonetos ${ }^{10,11}$, na redução de $\mathrm{NO}$ com $\mathrm{CO}^{12}$

e-mail: nagipe@uenf.br ou hidrogênio ${ }^{13}$ para produzir $\mathrm{CO}_{2} \mathrm{e} \mathrm{N}_{2}$. Outras aplicações, tais como combustão do metano ${ }^{14,15}$, acoplamento oxidativo de alcanos ${ }^{16}$, hidrogenação e hidrogenólise de hidrocarbonetos ${ }^{17,18}$ e oxidação da amônia ${ }^{19}$, evidenciam a importância dos óxidos do tipo perovskitas.

Vários trabalhos na literatura ${ }^{20,21}$ tratam do estudo da oxidação total de compostos orgânicos voláteis (COVs). Em trabalho recente, Spinicci et al. ${ }^{20}$ estudaram a oxidação de acetona, isopropanol e benzeno em presença das perovskitas $\mathrm{LaMnO}_{3}$ e $\mathrm{LaCoO}_{3}$. A atividade diminui da acetona ao benzeno na presença de ambos os catalisadores, embora o catalisador $\mathrm{LaMnO}_{3}$ seja o mais ativo. Esta maior atividade foi atribuída às espécies de oxigênio livre e com mobilidade suficiente. As cobaltitas e manganitas de lantânio têm sido igualmente aplicadas na destruição de compostos orgânicos voláteis clorados $(\mathrm{COVCs})^{22}$.

No passado, as aplicações das perovskitas como catalisadores foram muito limitadas, devido à obtenção de áreas superficiais muito pequenas, geralmente menor que $5 \mathrm{~m}^{2} / \mathrm{g}$. A principal razão da baixa área obtida deve-se ao fato que as perovskitas são essencialmente fases de óxidos obtidas a temperaturas elevadas e todos os métodos usados na preparação envolviam uma etapa de aquecimento pelo menos igual à essa temperatura. No caso de $\mathrm{LaCoO}_{3}$, esta temperatura pode chegar a $1073 \mathrm{~K}^{23}$. Mais recentemente, alguns autores citam a temperatura de 823 - $923 \mathrm{~K}$, no caso de preparação a partir de solução sol-gel ${ }^{24}$. Alguns exemplos de DRX demonstram que as perovskitas altamente cristalinas somente podem ser obtidas quando submetidas a calcinações a temperaturas superiores a $1073 \mathrm{~K}$. Um trabalho recente mostra que com o emprego do método de complexação de citratos, usando $\mathrm{ZnO}$ como aditivo solúvel, obtémse após calcinação à $973 \mathrm{~K}$, uma perovskita com boa cristalinidade ${ }^{23}$. Assim, a temperatura de calcinação é de grande importância na preparação dos óxidos do tipo perovskitas. De fato, o aumento desta temperatura resulta em um severo processo de sinterização, que provoca não somente um decréscimo da área superficial, mas também da densidade defeitos superficiais, esses provavelmente ativos como sítios catalíticos de oxidação/redução.

Na preparação das perovskitas são envolvidos métodos quími$\cos$ e físicos ${ }^{25}$, mediante reações envolvendo sólido - sólido e líquido - sólido. As reações do tipo sólido - sólido são usadas, principalmen- 
te, quando as áreas superficiais resultantes não constituem um parâmetro importante, na preparação de materiais cerâmicos (método cerâmico). Os métodos químicos líquidos - sólido requerem aparelhagens mais simples e podem originar áreas superficiais maiores que $40 \mathrm{~m}^{2} / \mathrm{g}$. Entre os métodos químicos podem-se citar cristalização, co-precipitação e complexação. Ainda sobre os métodos físicos, alguns autores constataram que a trituração de reagentes óxidos, seguida da adição de alguns aditivos, pode ser uma rota para obtenção, após calcinação a $473 \mathrm{~K}$, de perovskitas de elevadas áreas superficiais $^{26}$.

Alguns autores suportam as perovskitas em alguns suportes a fim de aumentar sua área específica. Assim, cobaltitas e manganitas de lantânio foram suportadas em óxido de zircônio, $\mathrm{ZnO}_{2}$, e examinadas na reação de combustão do metano e oxidação do $\mathrm{CO}^{10}$.

O método de preparação utilizado nesse trabalho foi desenvolvido por Barnard et al. ${ }^{27}$ e baseia-se na co-precipitação de sais (nitrato de cobalto e nitrato de lantânio) para obtenção de catalisadores de $\mathrm{LaCoO}_{3}$ com elevadas áreas superficicias. Neste trabalho, este método será igualmente empregado na preparação de catalisadores do tipo $\mathrm{LaMnO}_{3}$.

\section{PARTE EXPERIMENTAL}

\section{Preparação dos catalisadores}

Inicialmente, para a preparação das perovskitas à base de cobalto, foram preparadas as soluções dos sais de nitrato de cobalto II e nitrato de lantânio contendo $20 \mathrm{mmol}$ de cada um desses sais em $100 \mathrm{ml}$ de água destilada. A solução de nitrato de cobalto II foi preparada em laboratório pela interação do óxido de lantânio com ácido nítrico $6 \mathrm{M}$, seguido de aquecimento quase à secura e, conseqüentemente, adição de água destilada para obtenção da quantidade especificada anteriormente. Na preparação das perovskitas à base de manganês empregou-se, como sal fornecedor de cátions do tipo B, o cloreto de manganês (II). As soluções preparadas foram misturadas e colocadas em repouso por aproximadamente $1 \mathrm{~h}$.

Em outro recipiente, preparou-se uma solução oxidante de hidróxido de sódio (7,2 g) em hipoclorito de sódio (3,5 M, $30 \mathrm{ml})$.

Em seguida, sob atmosfera inerte de nitrogênio, adicionou-se gota-a-gota e sob constante agitação a solução de sais na mistura oxidante. A agitação requerida foi obtida através da passagem de um fluxo elevado de nitrogênio pela solução. O emprego da atmosfera inerte é necessário para minimizar a formação de carbonato do produto final. De fato, tem sido constatado que $\mathrm{CO}_{2}$ proveniente do ar atmosférico sorve-se facilmente sobre os géis de hidróxido de metais de terras raras ${ }^{27}$, formando carbonatos. O gel obtido foi lavado cerca de três vezes com $100 \mathrm{ml}$ de água deionizada de cada vez. A cada lavagem, o gel foi separado da fase líquida por filtração. Procedeu-se, então, à lavagem com acetona, aproximadamente $300 \mathrm{ml}$, sendo cada etapa de $100 \mathrm{ml}$. O material preparado foi seco à temperatura ambiente por $48 \mathrm{~h}$.

\section{Caracterização físico química}

\section{Difração de raios $X$}

A identificação da fase do catalisador foi conduzida em um difratômetro de raios X do tipo URD 65 - Rich Seifert \& Co. As condições usadas foram radiação $\mathrm{Cu} \mathrm{K \alpha}$, filtro $\mathrm{Ni}, 30 \mathrm{kV}, 40 \mathrm{~m} \mathrm{~A}$, varredura $0,05(2 \theta) / 5 \mathrm{~s}$. Os difratogramas obtidos foram comparados com os padrões do JCPDS.

\section{BET}

As medidas de fisissorção foram realizadas em um equipamento do tipo Autosorb 1c da Quantachrome. A área superficial BET foi determinada através da adsorção de nitrogênio a $77 \mathrm{~K}$ e pressão relativa na faixa de $0,05\left(\mathrm{p} / \mathrm{p}^{0}(0,20\right.$, considerando-se para a molécula de nitrogênio uma área de $\left.0,162 \mathrm{~nm}^{2}\right)$. Antes das medidas de adsorção as amostras foram desgaseificadas em vácuo de $10-6$ torr a $573 \mathrm{~K}$ por $1 \mathrm{~h}$.

\section{Análise térmica ATD - ATG}

As análises térmica diferencial (ATD) e térmica gravimétrica (ATG) foram realizadas em um equipamento Universal V2 6D TA Instruments. Os óxidos secos apenas à temperatura ambiente por 48 $\mathrm{h}$ foram tratados com fluxo de ar sintético por $15 \mathrm{~min}$, sendo depois aquecidos sob temperatura controlada $\left(10^{\circ} \mathrm{C} / \mathrm{min}\right)$ até $600{ }^{\circ} \mathrm{C}$.

\section{$X P S$}

A espectroscopia de fotoelétrons excitados por raios X (XPS), foi realizada em um espectrômetro VG Scientific Escalab Mark II com fonte de raios X Al K $\mathrm{K}_{\alpha}$. A pressão base na câmara de XPS foi menor que $1,0.10^{-9}$ torr. O sinal C $1 \mathrm{~s}$ em $284,9 \mathrm{eV}$ foi usado como referência para obter as energias de ligação.

\section{Análise química}

A valência do estado do manganês foi determinada pela dissolução da amostra em excesso conhecido de uma solução padrão de sulfato ferroso e pela titulação do $\mathrm{Fe}^{2+}$ em excesso com permanganato de potássio ${ }^{28}$.

\section{Teste catalítico}

Os catalisadores preparados e caracterizados foram testados nas reações de oxidação do propano e do $\mathrm{CO}$. As medidas de atividade foram realizadas em um microrreator de leito fixo operando em pressão atmosférica. $\mathrm{O}$ reator, um tubo vertical de $6 \mathrm{~mm}$ de diâmetro interno, era feito de quartzo e colocado em um forno elétrico também vertical com altura de $250 \mathrm{~mm}$. A temperatura do catalisador foi monitorada por um termopar de chromel - alumel associado a um programador linear de temperatura do tipo EDGON 3P. A alimentação do reator foi feita através de uma mistura padrão com a seguinte composição: $\mathrm{O}_{2}=2,02 \%, \mathrm{CO}=0,97 \%$ e $\mathrm{C}_{3} \mathrm{H}_{8}=0,507 \%$. O fluxo da mistura reacional foi de $1,8 \mathrm{l} / \mathrm{h}$.

Os produtos foram analisados em linha por cromatografia gasosa (Finnigam 9001) com detetor de ionização de chama para análise dos compostos orgânicos e coluna do tipo Porapack Q de $3 \mathrm{~m}$ de comprimento e $1 / 8$ de polegadas de diâmetro, utilizando nitrogênio como gás de arraste. O dióxido de carbono proveniente da oxidação do propano e CO foi monitorado através de um analisador FTIR (Hartmann and Braun URAS 14).

\section{RESULTADOS E DISCUSSÃO}

Na preparação dos óxidos tipo perovskitas, $\mathrm{LaCoO}_{3}$ e $\mathrm{LaMnO}_{3}$, foi empregado um método de co-precipitação - oxidação, desenvolvido anteriormente ${ }^{27}$ para preparação de catalisadores $\mathrm{LaCoO}_{3}$.

A vantagem deste método em relação a outros, em que a desidratação do gel formado é realizada apenas com aquecimento em presença de ar, é o fato de ser feita uma desidratação inicial em presença de acetona. Tal desidratação controlada de hidrogéis, usando água e um solvente miscível, já mostrou resultados positivos na obtenção de sólidos com elevadas áreas superficiais ${ }^{29,30}$. O deslocamento da água pela acetona em tal gel, permite uma diminuição da tensão superficial e origina menor força capilar, produzindo aglomerações mais fracas e mantendo a estrutura das partículas do hidróxido, sob aquecimento, mais aberta. $\mathrm{O}$ resultado é um menor colapso durante a calcinação $0^{30}$. As melhores medidas de área superficial foram encon- 
tradas após as amostras serem calcinadas inicialmente a $400{ }^{\circ} \mathrm{C}$ por $1 \mathrm{~h}$ e, em seguida, a $600{ }^{\circ} \mathrm{C}$ por mais $1 \mathrm{~h}$. Entretanto, alterando as temperaturas de calcinação e eventualmente a lavagem com acetona, vários materiais catalíticos foram preparados.

Os resultados da análise térmica sobre os catalisadores podem ser acompanhados através das Figuras 1 e 2. Os termogramas para as supostas perovskitas à base de cobalto e manganês apresentam picos endotérmicos à baixa temperatura, correspondendo à eliminação de água fisissorvida. Este resultado está de acordo com o termograma de ATG, onde a perda de massa nos dois casos corresponde a $2,7 \%$ no caso da cobaltita de lantânio e $1,16 \%$ no caso da manganita de lantânio. Em aproximadamente $550{ }^{\circ} \mathrm{C}$, o termograma ATD relativo à cobaltita apresenta um pico exotérmico. Já no caso da manganita, um pico exotérmico de menor intensidade foi observado em aproximadamente $720{ }^{\circ} \mathrm{C}$. Esses picos podem ser associados com a cristalização das perovskitas. Este fato pode ser comprovado pelo termograma ATG, onde se observa nessas temperaturas uma queda brusca de peso proveniente provavelmente da liberação de $\mathrm{CO}_{2}$, oriundo da decomposição de carbonato formado durante a preparaçã $0^{28}$.

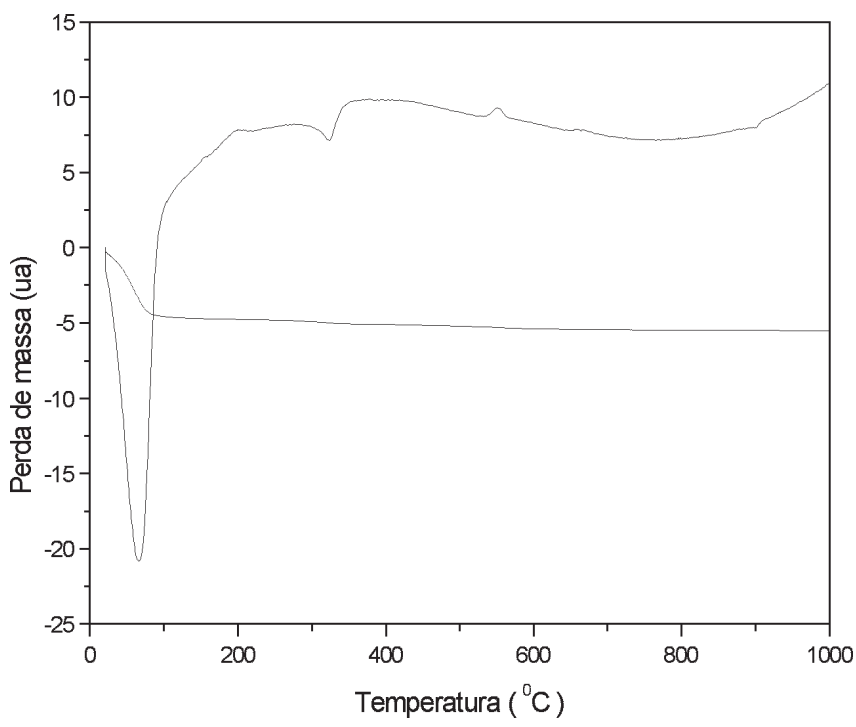

Figura 1. Termograma ATG - ATD da cobaltita de lantânio

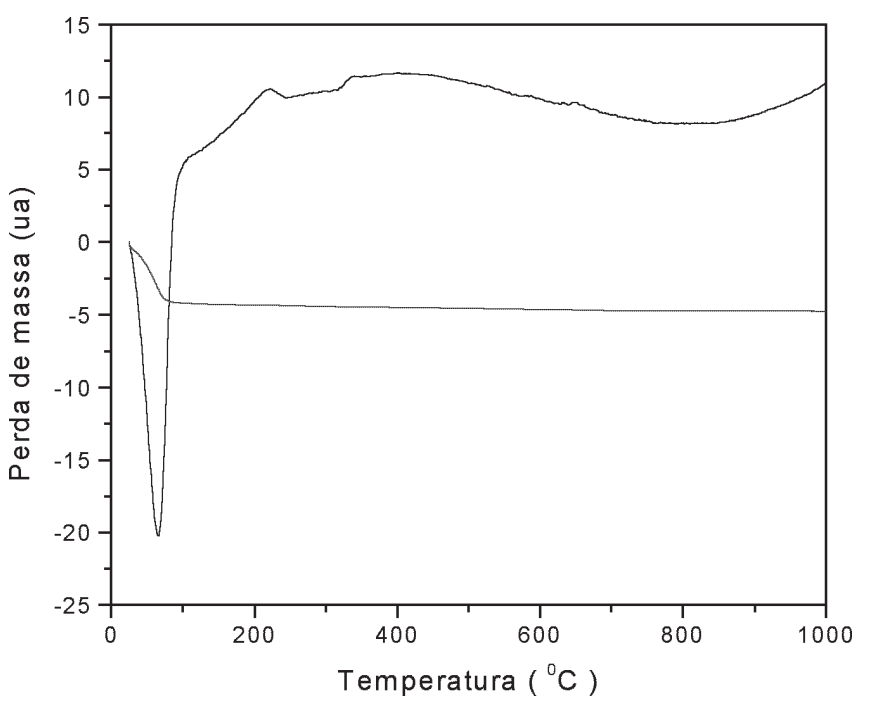

Figura 2. Termograma ATG - ATD da manganita de lantânio
Após desidratação à $673 \mathrm{~K}$ por $1 \mathrm{~h}$ e calcinação à $873 \mathrm{~K}$ por igual tempo, as amostras foram submetidas à análise de DRX. O modelo de difração obtido para o catalisador $\mathrm{LaCoO}_{3}$ corresponde à estequiometria da perovskita descrita no JCPDS: 25-1060.

O modelo DRX para o catalisador à base de manganês - lantânio, é mais próximo da estrutura $\mathrm{LaMnO}_{3}$ não estequiométrica, com excesso de oxigênio (JCPDF: 32-0484), a qual apresenta uma simetria ortorrômbica. Convém ressaltar que este fator por si só não comprova a existência de tal estrutura. À baixa temperatura, a estrutura romboédrica de $\mathrm{LaMnO}_{3}$ é, sem dúvida, rica em vacâncias catiônicas ${ }^{3}$, o que corresponde a um excesso de oxigênio com relação à estequiometria $(\delta=0,1-0,2)$, acompanhado pela transição espontânea de $15-30 \%$ de $\mathrm{Mn}^{3+}$ a $\mathrm{Mn}^{4+}$ para restabelecer a eletroneutralidade do sistema. De outro lado, além dos picos característicos da perovskita $\mathrm{LaMnO}_{3}$, observam-se picos característicos de $\mathrm{LaOCl}$ (Figura 4), aqui representados por (*), JCPDS 34-1494, indicando a presença de traços do referido óxido. A presença de $\mathrm{LaOCl}$ já foi constatada por outros autores ${ }^{22}$, onde o efeito do cloro foi estudado sobre os dois tipos de catalisadores.

A caracterização da superfície dos catalisadores $\mathrm{LaCoO}_{3} \mathrm{e} \mathrm{LaMnO}_{3}$ foi realizada por análise XPS com a utilização de C1s como referência. A Tabela 1 lista os valores das energias de ligação correspondentes para: O 1s, La 3d, Co 2p e Mn 2p. Vários trabalhos têm demonstrado que a maioria dos cátions está presente na forma trivalente ${ }^{31}$.

Segundo a análise dos dados para $\mathrm{LaCoO}_{3}$ e referentes ao $\mathrm{La} 3 \mathrm{~d}$ $5 / 2$ são registrados em 835,3 e $838,8 \mathrm{eV}$, sendo muito semelhantes aos valores apresentados na literatura ${ }^{22}$ para o óxido de lantânio puro $\left(\mathrm{La}_{2} \mathrm{O}_{3}\right)$ em 834,4 e 837,8 eV. Esses dados indicam que o lantânio provavelmente está presente na forma trivalente.

Os sinais em 779,9 e 795,5 eV correspondem ao Co $2 \mathrm{p}_{3 / 2}$ e Co $2 \mathrm{p}_{1 / 2}$, respectivamente, típicos da presença de $\mathrm{Co}^{3+}$. De outro lado, a ausência de picos satélites na região de $785-788 \mathrm{eV}$ é prova da inexistência de $\mathrm{Co}^{2+}$. Isto comprova a teoria sobre a existência dos cátions em suas formas trivalentes.

Espectro XPS do catalisador $\mathrm{LaCoO}_{3}$ (Figura 3) apresenta a deconvolução do pico $\mathrm{O} 1 \mathrm{~s}$ em dois picos, que correspondem a duas espécies de oxigênio. $\mathrm{O}$ pico de menor energia, $529,8 \mathrm{eV}$, pode ser associado com o oxigênio da rede, enquanto o de maior energia, $532,5 \mathrm{eV}$, pode ser associado a espécies de oxigênio absorvido, embora esta fração contenha também oxigênio adsorvido. A fração do oxigênio absorvido - adsorvido podendo ser representado pelas espécies $\mathrm{O}^{-}$e $\mathrm{OH}^{-32}$.

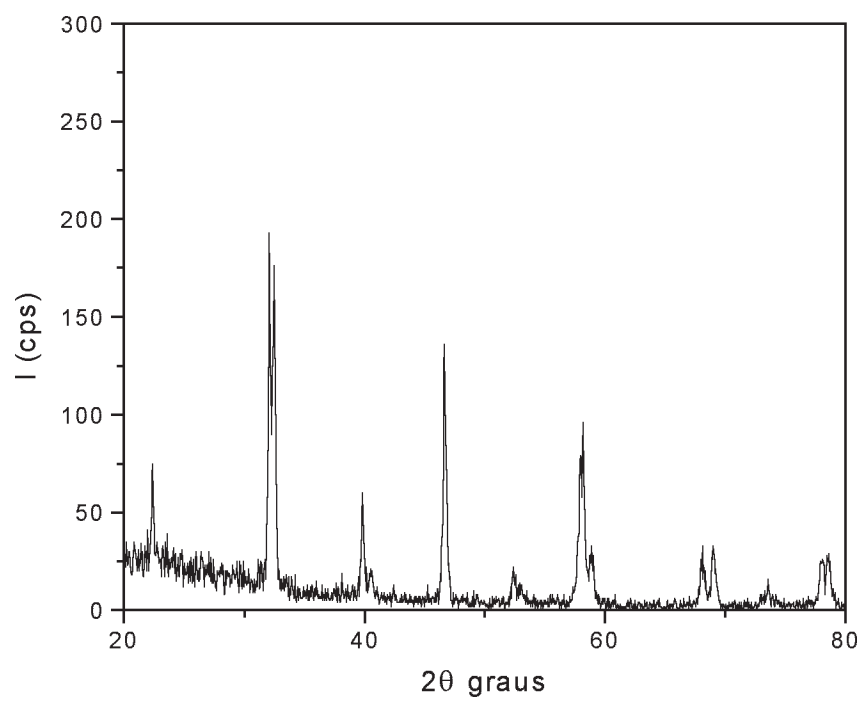

Figura 3. Difratograma de raios $\mathrm{X}$ da amostra $\mathrm{LaCoO}_{3}$ 
$\mathrm{Na}$ literatura ${ }^{32}$, os valores de energia correspondentes a espécie de oxigênio $\mathrm{O}^{-}$aparecem em 528,3 e 531,9 eV. O primeiro representa o oxigênio da rede e o segundo, a parte absorvida. Já no caso da espécie OH-, esses valores passam a 529,7 e 532,2 eV. Convém ressaltar a semelhança dos resultados de energia de ligação para a espécie $\mathrm{OH}^{-}$com os apresentados nesse trabalho, indicando maior probabilidade da existência do mesmo.

No caso do catalisador $\mathrm{LaMnO}_{3}$ (Figura 4), todos os picos são deslocados de aproximadamente $6 \mathrm{eV}$ em relação aos valores de energia de ligação encontrados no espectro do catalisador $\mathrm{LaCoO}_{3}$. Isto pode estar relacionado à presença de cloro, conforme indicou a presença da fase $\mathrm{La} \mathrm{O} \mathrm{Cl} \mathrm{na} \mathrm{difração} \mathrm{de} \mathrm{raios} \mathrm{X.} \mathrm{Assim,} \mathrm{picos} \mathrm{referentes}$ ao La 3d 5/2 são deslocados para região de maior valor de energia de ligação $(841,10$ e $845 \mathrm{eV})$. Um único pico correspondente a $\mathrm{O} 1 \mathrm{~s}$ aparece somente em 536,60 eV. A ausência do pico na região de maior energia indica apenas o envolvimento do oxigênio da rede. Alterações na estrutura cristalina do composto e também na sua estrutura eletrônica podem ser as responsáveis pelo deslocamento citado anteriormente.

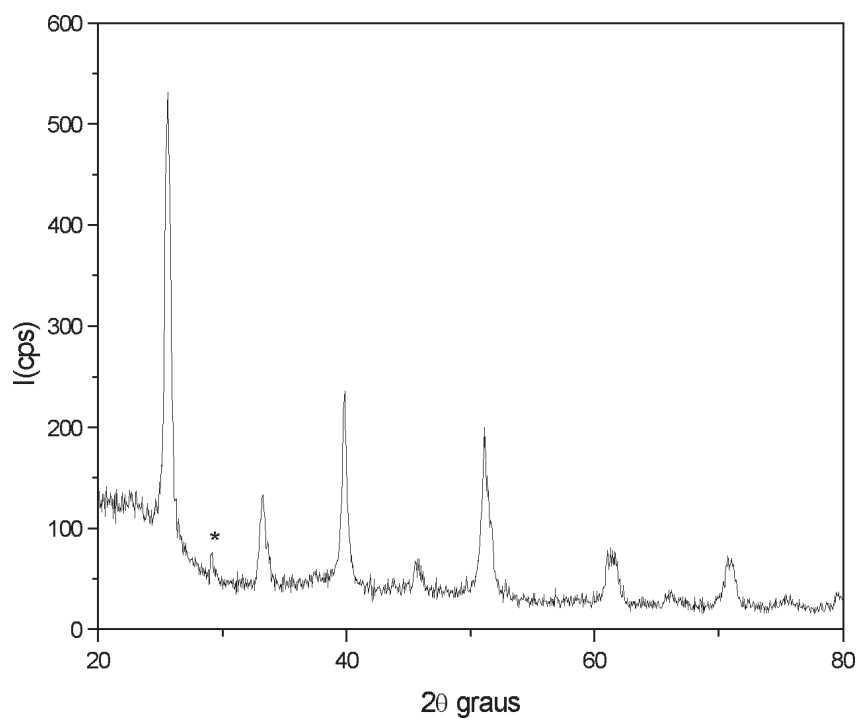

Figura 4. Difratograma de raios $\mathrm{X}$ da amostra $\mathrm{LaMnO}_{3}$

$\mathrm{O} \mathrm{Mn} 2 \mathrm{p}$ apresenta três sinais correspondendo a Mn3+ $(647,40 \mathrm{eV}), \mathrm{Mn} 4+(658,7 \mathrm{eV})$ e à presença de um pico satélite, indicando a ausência de $\mathrm{Mn}^{2+}$. A presença de cloro é constatada através de um pico bem definido em aproximadamente $274,0 \mathrm{eV}$.

No que se refere ao nível C 1s, observa-se, embora ainda pequeno, um grau maior de contaminação por carbonato da superfície de manganita em $289 \mathrm{eV}$.

É também conveniente comparar as informações da estequiometria obtida pelas medidas de XPS (Tabela 1). A razão atômica La /Co na superfície é de 1,8. Isto sugere a presença de excesso de lantânio ou deficiência de Co na superfície da cobaltita $\mathrm{LaCoO}_{3}$. Alguns autores têm observado a presença de excesso de lantânio nas estruturas das perovskitas $(\mathrm{La} / \mathrm{Co}>1)$. Tabata et al.${ }^{33}$ mencionaram a razão $\mathrm{La} / \mathrm{Co}$ como sendo 1,2 no caso de catalisadores $\mathrm{LaCoO}_{3}$, obtidos pela síntese com acetato. $\mathrm{O}$ caso mais pronunciado foi observado por Connell et al. ${ }^{31}$, que prepararam a cobaltita através da precipitação da soluções de nitrato de cobalto e lantânio, com hidróxido de tetraetilamônio. Para este caso a razão La/Co foi 4,26, evidenciando a presença de um grande excesso de lantânio na superfície. Este excesso de lantânio na superfície foi atribuído à formação de duas fases; uma correspondendo à perovskita pura e a outra formada por
La/perovskita, ambas com deficiência em íons lantânio. Na primeira, as vacâncias catiônicas são compensadas pela presença das valências elevadas de cobalto e oxigênio; na segunda, pela presença de vacâncias aniônicas. Esta última sendo, cataliticamente, muito mais interessante.

De uma maneira geral, muitos autores têm considerado o sistema $\mathrm{LaCoO}_{3}$ como estequiométrico. De outro lado, o sistema é apontado como não estequiométrico como é o caso do trabalho de Wu et $a l .{ }^{34}$. Nos sistemas não estequiométricos geralmente existe uma grande deficiência de lantânio na rede, que pode ser compensada pelo aumento na valência do metal do tipo B, ou do oxigênio ou, ainda, pela criação de vacâncias aniônicas.

Em nosso caso, pode-se observar que a quantidade de íons lantânio é quase o dobro dos íons cobalto. Assim, poder-se-ia supor primeiramente a formação de uma cobaltita de lantânio estequiométrica, juntamente com outros compostos à base de lantânio como, por exemplo, óxido de lantânio. Entretanto, através da difração de raios $\mathrm{X}$ não foi observada outra fase que as da perovskita, descartando a hipótese de formação de uma perovskita estequiométrica. Assim, já que a perovskita é não estequiométrica e não há formação de outros compostos, a razão encontrada provavelmente é devido à deficiência em cobalto.

De outro lado, a razão La/Mn é de 0,79 , sugerindo que a fase da manganita de lantânio não é estequiométrica e sim, com deficiência em lantânio. Para manter o equilíbrio de cargas, a seguinte conversão pode ser considerada; $\mathrm{Mn}^{3+} \rightarrow \mathrm{Mn}^{4+}$. Esta transição é acompanhada pela geração de vacâncias catiônicas e pela presença de excesso de oxigênio. Tagauchi et al. $^{35}$ encontraram para o catalisador $\mathrm{LaMnO}_{3}$, dependendo do método de preparação, razões La/Mn que variavam de 1,5 - 2. Entretanto, parte do lantânio não estava incorporada à perovskita, mas se apresentava na forma de óxidos, onde parte desses óxidos estava carbonatada.

A razão oxigênio-íon metálico total $(\mathrm{O} / \mathrm{La}+\mathrm{Co})$ no catalisador $\mathrm{LaCoO}_{3}$ é aproximadamente igual a 3,5, em perfeito acordo com o resultado encontrado por Connell et al. ${ }^{26}$. Já no catalisador $\mathrm{LaMnO}_{3}$, esta razão é de aproximadamente 2,5, ambas superiores à razão de 1,5, esperada caso a estequiometria fosse obedecida. Isto comprova que esses materiais possuem excesso de oxigênio. Este resultado comprova que o excesso de oxigênio no caso da cobaltita e da manganita caracteriza a formação de vacâncias catiônicas. Logo, estamos diante uma cobaltita com deficiência em cobalto e de uma manganita com deficiência em manganês. Entretanto, a supremacia da quantidade de oxigênio não estequiométrico da cobaltita em relação à manganita, pode indicar a presença de maior quantidade de vacâncias catiônicas, no primeiro caso. Além disso, no caso da manganita parte dessas vacâncias é minimizada pela transição $\mathrm{Mn}^{3+} \rightarrow \mathrm{Mn}^{4+}$.

Na Tabela 1 são apresentados os resultados de XPS (energia de ligação e razão atômica) em relação aos elementos presentes na constituição dos catalisadores.

Tabela 1. Resultados de XPS para as perovskitas

\begin{tabular}{ccc}
\hline Nível & $\mathrm{LaCoO}_{3}[\mathrm{eV}]$ & $\mathrm{LaMnO}_{3}[\mathrm{eV}]$ \\
\hline $\mathrm{O}(1 \mathrm{~s})$ & 529,8 & \\
& 532,4 & - \\
$\mathrm{La}(3 \mathrm{~d})$ & 835,3 & 841,10 \\
& 838,8 & 845,0 \\
$\mathrm{Co}(2 \mathrm{p})$ & 779,9 & - \\
& 795,5 & - \\
$\mathrm{Mn}$ & - & 649,10 \\
& & 660,10 \\
$\mathrm{O} / \mathrm{La}$ & 5,234 & 5,732 \\
$\mathrm{Co} / \mathrm{La}$ & 0,550 & - \\
$\mathrm{Mn} / \mathrm{La}$ & - & 1,260 \\
\hline
\end{tabular}


As atividades dos catalisadores preparados foram medidas através das seguintes reações:

$\mathrm{CO}+1 / 2 \mathrm{O}_{2} \rightarrow \mathrm{CO}_{2}$

$\mathrm{C}_{3} \mathrm{H}_{8}+5 \mathrm{O}_{2} \rightarrow 3 \mathrm{CO}_{2}+4 \mathrm{H}_{2} \mathrm{O}$

A primeira reação, oxidação do $\mathrm{CO}$, ocorre a temperaturas mais baixas e a segunda, oxidação total do propano, a temperaturas mais elevadas.

Os resultados obtidos na reação de oxidação do propano podem ser vistos nas Tabelas 2 e 3 . Nessas tabelas pode-se comparar a variação da conversão com a temperatura e a área total dos catalisadores. Pode-se observar que a conversão aumenta à medida que aumenta a área superficial total e que as perovskitas $\mathrm{LaCoO}_{3}$ são mais ativas que as $\mathrm{LaMnO}_{3}$ exigindo, desta forma, menor temperatura na obtenção de um mesmo grau de conversão. Para melhor comparação, podem-se comparar catalisadores que apresentem áreas totais próximas ou semelhantes; como é o caso da cobaltita, $\mathrm{LaCoO}_{3}-\mathrm{V}$ com área de $34,0 \mathrm{~m}^{2} / \mathrm{g}$ e a manganita, $\mathrm{LaMnO}_{3}-\mathrm{I}, 39,0 \mathrm{~m}^{2} / \mathrm{g}$. Nota-se que no caso da cobaltita é preciso uma temperatura de $148{ }^{\circ} \mathrm{C}$ para obtenção de uma conversão de $5,0 \%$, enquanto no caso da manganita é preciso uma temperatura de $308^{\circ} \mathrm{C}$ para obtenção do mesmo grau de conversão.
De outro lado, a cobaltita atinge a conversão máxima em temperatura de $382{ }^{\circ} \mathrm{C}$, enquanto a manganita atinge a mesma conversão a $458^{\circ} \mathrm{C}$. Convém ressaltar que esta diferença é mais pronunciada em temperaturas mais baixas.

No caso da reação de oxidação do $\mathrm{CO}$, os resultados podem ser vistos nas Tabelas 4 e 5 , onde as cobaltitas continuam sendo mais ativas. Os catalisadores $\mathrm{LaCoO}_{3}-\mathrm{V}$ e o $\mathrm{LaMnO}_{3}$ - I convertem $5 \%$ de $\mathrm{CO}$ nas temperaturas de 122 e $140{ }^{\circ} \mathrm{C}$, respectivamente. De outro lado, eles atingem a conversão máxima em 160 e $320^{\circ} \mathrm{C}$, respectivamente. Aqui as diferenças de atividade são mais importantes nas temperaturas mais elevadas, já que a reação de oxidação de CO ocorre em temperaturas mais baixas. A excelente atividade das cobaltitas de lantânio na reação de oxidação do CO foi ressaltada por Shu et al.$^{25}$.

Existem várias discussões sobre as atividades das perovskitas. Dentre as menos comuns destacam-se: estados de oxidação de íons de metais de transição; a quantidade de oxigênio não estequiométrico e a estrutura de defeitos da rede.

Aparece freqüentemente na literatura que o oxigênio não estequiométrico pode ser um fator de considerável importância na interpretação da atividade catalítica de uma série de perovskitas de metais de transição, inclusive com a participação de promotores ${ }^{28}$. Entretanto, a estrutura química da rede de uma perovskita é muito complexa, podendo haver distorções do tipo hexagonal, romboédrica

Tabela 2. Conversão de propano em $\mathrm{CO}_{2}$ em função da área total e da temperatura para as cobaltitas

\begin{tabular}{|c|c|c|c|c|c|}
\hline Catalisadores & $\mathrm{LaCoO}_{3}-\mathrm{I}$ & $\mathrm{LaCoO}_{3}-\mathrm{II}$ & $\mathrm{LaCoO}_{3}$-III & $\mathrm{LaCoO}_{3-} \mathrm{IV}$ & $\mathrm{LaCoO}_{3}-\mathrm{V}$ \\
\hline Área $\mathrm{m}^{2} / \mathrm{g}$ & 12,0 & 22,0 & 27,0 & 30,0 & 34,0 \\
\hline$\%$ de conversão & $\mathrm{T} /{ }^{\circ} \mathrm{C}$ & $\mathrm{T} /{ }^{\circ} \mathrm{C}$ & $\mathrm{T} /{ }^{\circ} \mathrm{C}$ & $\mathrm{T} /{ }^{\circ} \mathrm{C}$ & $\mathrm{T} /{ }^{\circ} \mathrm{C}$ \\
\hline 5,0 & 186 & 180 & 166 & 160 & 148 \\
\hline 10,0 & 203 & 190 & 180 & 176 & 161 \\
\hline 15,0 & 221 & 201 & 193 & 188 & 173 \\
\hline 25,0 & 255 & 242 & 221 & 212 & 198 \\
\hline 30,0 & 273 & 263 & 234 & 225 & 210 \\
\hline 40,0 & 307 & 304 & 262 & 249 & 235 \\
\hline 50,0 & 342 & 332 & 289 & 273 & 259 \\
\hline 60,0 & 377 & 372 & 316 & 297 & 284 \\
\hline 70,0 & 412 & 406 & 344 & 321 & 309 \\
\hline 80,0 & 446 & 440 & 371 & 345 & 333 \\
\hline 90,0 & 481 & 472 & 398 & 369 & 358 \\
\hline 100,0 & 516 & 503 & 426 & 394 & 382 \\
\hline
\end{tabular}

Tabela 3. Conversão de propano a $\mathrm{CO}_{2}$ em função da área total e da temperatura para as manganitas

\begin{tabular}{|c|c|c|c|c|c|}
\hline Catalisadores & $\mathrm{LaMnO}_{3}-\mathrm{I}$ & $\mathrm{LaMnO}_{3}-\mathrm{II}$ & $\mathrm{LaMnO}_{3}$-III & $\mathrm{LaMnO}_{3}-\mathrm{IV}$ & $\mathrm{LaMnO}_{3}-\mathrm{V}$ \\
\hline Área $\mathrm{m}^{2} / \mathrm{g}$ & 39,0 & 44,0 & 55,0 & 59,0 & 60,0 \\
\hline$\%$ de conversão & $\mathrm{T} /{ }^{\circ} \mathrm{C}$ & $\mathrm{T} /{ }^{\circ} \mathrm{C}$ & $\mathrm{T} /{ }^{\circ} \mathrm{C}$ & $\mathrm{T} /{ }^{\circ} \mathrm{C}$ & $\mathrm{T} /{ }^{\circ} \mathrm{C}$ \\
\hline 5,0 & 308 & 252 & 259 & 228 & 216 \\
\hline 10,0 & 316 & 262 & 266 & 237 & 225 \\
\hline 15,0 & 324 & 271 & 274 & 247 & 234 \\
\hline 25,0 & 340 & 290 & 289 & 265 & 252 \\
\hline 30,0 & 347 & 300 & 297 & 275 & 261 \\
\hline 40,0 & 363 & 319 & 312 & 293 & 279 \\
\hline 50,0 & 379 & 338 & 328 & 312 & 298 \\
\hline 60,0 & 395 & 357 & 343 & 331 & 316 \\
\hline 70,0 & 410 & 376 & 358 & 349 & 340 \\
\hline 80,0 & 426 & 395 & 373 & 368 & 352 \\
\hline 90,0 & 442 & 414 & 389 & 389 & 370 \\
\hline 100,0 & 456 & 433 & 414 & 405 & 388 \\
\hline
\end{tabular}


Tabela 4. Conversão de $\mathrm{CO}$ a $\mathrm{CO}_{2}$ em função da área total e da temperatura para as cobaltitas

\begin{tabular}{|c|c|c|c|c|c|}
\hline Catalisadores & $\mathrm{LaCoO}_{3}-\mathrm{I}$ & $\mathrm{LaCoO}_{3}$-II & $\mathrm{LaCoO}_{3}$-III & $\mathrm{LaCoO}_{3}$-IV & $\mathrm{LaCoO}_{3}-\mathrm{V}$ \\
\hline Área $\mathrm{m}^{2} / \mathrm{g}$ & 12,0 & 22,0 & 27,0 & 30,0 & 34,0 \\
\hline$\%$ de conversão & $\mathrm{T} /{ }^{\circ} \mathrm{C}$ & $\mathrm{T} /{ }^{\circ} \mathrm{C}$ & $\mathrm{T} /{ }^{\circ} \mathrm{C}$ & $\mathrm{T} /{ }^{\circ} \mathrm{C}$ & $\mathrm{T} /{ }^{\circ} \mathrm{C}$ \\
\hline 5,0 & 167 & 144 & 135 & 130 & 122 \\
\hline 10,0 & 170 & 147 & 138 & 133 & 124 \\
\hline 15,0 & 173 & 150 & 140 & 136 & 126 \\
\hline 25,0 & 178 & 156 & 145 & 141 & 130 \\
\hline 30,0 & 181 & 159 & 147 & 144 & 132 \\
\hline 40,0 & 186 & 166 & 152 & 149 & 136 \\
\hline 50,0 & 192 & 172 & 157 & 154 & 140 \\
\hline 60,0 & 197 & 178 & 162 & 160 & 144 \\
\hline 70,0 & 202 & 184 & 167 & 165 & 148 \\
\hline 80,0 & 208 & 191 & 172 & 171 & 152 \\
\hline 90,0 & 213 & 197 & 177 & 176 & 160 \\
\hline 100.0 & 219 & 203 & 181 & 182 & 160 \\
\hline
\end{tabular}

Tabela 5. Conversão de $\mathrm{CO}$ em $\mathrm{CO}_{2}$ em função da área total e da temperatura para as manganitas

\begin{tabular}{|c|c|c|c|c|c|}
\hline Catalisadores & $\mathrm{LaMnO}_{3}-\mathrm{I}$ & $\mathrm{LaMnO}_{3}$-II & $\mathrm{LaMnO}_{3}$-III & $\mathrm{LaMnO}_{3}-\mathrm{IV}$ & $\mathrm{LaMnO}_{3}-\mathrm{V}$ \\
\hline Área $\mathrm{m}^{2} / \mathrm{g}$ & 39,0 & 44,0 & 55,0 & 59,0 & 66,0 \\
\hline$\%$ de conversão & $\mathrm{T} /{ }^{\circ} \mathrm{C}$ & $\mathrm{T} /{ }^{\circ} \mathrm{C}$ & $\mathrm{T} /{ }^{\circ} \mathrm{C}$ & $\mathrm{T} /{ }^{\circ} \mathrm{C}$ & $\mathrm{T} /{ }^{\circ} \mathrm{C}$ \\
\hline 5,0 & 149 & 146 & 144 & 143 & 137 \\
\hline 10,0 & 158 & 153 & 151 & 149 & 142 \\
\hline 15,0 & 167 & 159 & 158 & 154 & 147 \\
\hline 25,0 & 185 & 172 & 172 & 165 & 156 \\
\hline 30,0 & 194 & 178 & 178 & 171 & 161 \\
\hline 40,0 & 213 & 191 & 192 & 181 & 170 \\
\hline 50,0 & 231 & 204 & 205 & 192 & 180 \\
\hline 60,0 & 249 & 216 & 219 & 203 & 189 \\
\hline 70,0 & 267 & 229 & 233 & 214 & 197 \\
\hline 80,0 & 286 & 242 & 246 & 225 & 208 \\
\hline 90,0 & 304 & 255 & 260 & 236 & 217 \\
\hline 100,0 & 322 & 267 & 273 & 247 & 227 \\
\hline
\end{tabular}

e tetragonal. Esta distorção do sistema cúbico pode resultar em uma não estequiometria catiônica ou aniônica.

A oxidação de monóxido de carbono sobre óxidos do tipo perovskita tem sido muito estudada. A partir de dados cinéticos e dados da espectroscopia infravermelho, foi proposto um esquema para a oxidação do $\mathrm{CO}$ sobre $\mathrm{LaCoO}_{3}^{2}$ :

$\mathrm{O}_{2}(\mathrm{~g}) \rightarrow \mathrm{O}_{2}$ (ads) $\rightarrow 2 \mathrm{O}$ (ads.)

$\mathrm{CO}(\mathrm{g}) \rightarrow \mathrm{CO}$ (ads.)

$\mathrm{CO}($ ads $)+2 \mathrm{O}$ (ads) $\rightarrow \mathrm{CO}_{3}$ (ads.) lenta

$\mathrm{CO}_{3}$ (ads) $\rightarrow \mathrm{CO}_{2}$ (ads) $+\mathrm{O}$ (ads.)

$\mathrm{CO}_{2}$ (ads) $\rightarrow \mathrm{CO}_{2}(\mathrm{~g})$

Onde a etapa (5) seria a etapa determinante da velocidade. Oxigênio é adsorvido como espécie $\mathrm{O}_{2}^{-}$sobre íons cobalto de baixo estado de oxidação, o qual subseqüentemente dissocia-se, originando espécies de oxigênio atômico $\left(\mathrm{O}^{-}\right)$, adsorvido sobre o mesmo centro (2). $\mathrm{CO}$ é adsorvido sobre íons $\mathrm{O}^{2-}$ da rede, produzindo espécies lábeis que interagem com oxigênio atômico adsorvido, produzindo carbonato que se decompõe produzindo $\mathrm{CO}_{2}$ e oxigênio (2). Este resultado está em perfeito acordo com o resultado de análise térmica apresentado pela amostra de $\mathrm{LaCoO}_{3}$ nesse trabalho, onde a decom- posição do carbonato ocorre em aproximadamente $550{ }^{\circ} \mathrm{C}$. Para o caso do propano, poderíamos propor um mecanismo semelhante ao anterior, diferindo apenas na etapa inicial, onde iria gerar monóxido de carbono e água.

A manganita de lantânio, apesar de ser menos ativa que o catalisador $\mathrm{LaCoO}_{3}$, possui uma atividade intrínseca considerável devido à tendência do $\mathrm{Mn}^{+3}$ oxidar-se sob aquecimento em ar, à pressão atmosférica. A transição é acompanhada pela geração de vacâncias catiônicas e pela presença de um conhecido excesso de oxigênio em relação à estequiométrica necessária. $\mathrm{O}$ não aparecimento do pico exotérmico nas proximidades de $550{ }^{\circ} \mathrm{C}$ pode indicar que o mecanismo de oxidação e o processo de cristalização do $\mathrm{LaMnO}_{3}$ são diferentes dos apresentados para o catalisador $\mathrm{LaCoO}_{3}$.

\section{CONCLUSÃO}

Através do método de preparação empregando a co-precipitação de sais em meio oxidante consegue-se preparar cobaltitas e manganitas de lantânio, não estequiométricas, com consideráveis áreas superficiais e com grau relativamente baixo de contaminação por carbonato. Essas perovskitas são ricas em vacâncias catiônicas, que correspondem a um excesso de oxigênio em relação à estequiometria. Este 
excesso foi mais pronunciado no caso das cobaltitas, o que pode ser o causador de sua maior atividade nas reações de oxidação de $\mathrm{CO}$ e propano.

\section{AGRADECIMENTOS}

À FAPERJ e à FINEP/CTPETRO pelo apoio financeiro. Aos técnicos M. V. Rocha e R. N. Agum, ambos bolsistas, FINEP/ CTPETRO, pela ajuda na realização da caracterização das amostras.

\section{REFERÊNCIAS}

1. Tejuca, L. G.; Fierro, J. L. G. Em Properties and Applications of Perovskites - Type Oxides, Dekker, M., ed.; New York, 1993.

2. Tejuca, L. G.; Fierro, J. L. G.; Tascon, J. M. D.; Adv. Catal. 1989, 36, 237.

3. Misusaki, J.; Takai, N.; Yonemura, H.; Minamiue, Y.; Tagawa, H.; Dokiya, T.; Inaba M.; Naraya, H.; Sasamoto, K.; Hashimoto, T.; Solid State Ionics 2000, 129, 163

4. Kindermann, L.; Das, D.; Nickel, H.; Hilpert, K.; Solids State Ionics 1996, $89,215$.

5. Isuprova, L. A.; Tsybulya, S. V.; Kryukoya, G. N.; Alikina, G. M.; Boldyreva, N. N.; Yakovleva, I. S.; Ivanov, V. P.; Sadykov, V. A.; Solid State Ionics 2001, 141, 417.

6. Inaba, H.; Hayasashi, H.; Suzuki, M.; Solid State Ionics 2001, 144, 99.

7. Rojas, M. L.; Fierro, J. L. G.; Tejuca, L. G.; Bell, A. T.; J. Catal. 1990, $124,41$.

8. Furusaki, A.; Konno, H.; Furuichi, R.; J. Mater. Sci. 1995, 2829

9. Hansen, K. K.; Skou, E. M.; Chritensen, H.; Turek, T.; J. Catal. 2001, 199, 132.

10. Cimino, S.; Colonna, S.; De Rossi, S.; Faticanti, M.; Lisi, L.; Pettiti, I.; Porta, P.; J. Catal. 2002, 205, 309.

11. Song, K. S.; Klvana, D.; Kichnerova, J.; Appl. Catal. 2001, 213, 113.

12. Forni, L.; Oliva, C.; Barzetti, T.; Selli, E. A. M.; Vishiniakov, A.V.; Appl. Catal., B 1997, 13, 35 .
13. Ferri, D.; Forni, L.; Dekkers, M. A. P.; Nieuwenhuys, B. E.; Appl. Catal., $B$ 1998, 16, 339.

14. Nguyen, S. V.; Szabo, V.; Trong On, D.; Kaliaguine, S.; Microporous Mesoporous Mater. 2002, 54, 51.

15. Saracco, G.; Geobaldo, F.; Baldi, G.; Appl. Catal., B 1999, 20, 277.

16. Buyevskaya, O. V.; Rothaemel, M.; Zanthoff, H. W.; Baerns, M.; J. Catal. 1994, 71, 150 .

17. Libby, W. F.; Science 1971, 171, 499.

18. Pederson, L. A.; Libby, W. F.; Science 1972, 176, 1355.

19. Yu, Z.; Gao, L.; Yuan, S.; Wu, Y.; J. Chem. Soc., Faraday Trans. 1992, 88, 3245.

20. Spinicci, R.; Faticanti, M.; Marini, P.; De Rossi, S.; Porta, P.; J. Mol. Catal. 2003, 197, 147.

21. Spinnici, R.; Tofannari, A.; Faticanti, M.; Pettiti, I.; Porta, P.; J. Mol. Catal. 2001, 176, 247.

22. Sinquin, G.; Petit, C.; Hindermann, K.; Catal. Today 2001, 70, 183.

23. Crespin, M.; Hal, K. W.; J. Catal. 1981, 69.

24. Xiong, G.; Zhi, Z. L.; Yang, X. J.; Lu, L.; Wang, X.; J. Mater. Sci. Lett. 1997, 16, 1064.

25. Shu, J.; Kaliaguine, S.; Appl. Catal., B 1998, 16, 303.

26. Kaliaguine, S.; Van Neste, A.; Szabo, V.; Gallot, J. E.; Bassir, M.; Muzychuk, R.; Appl. Catal., A 2001, 209, 345.

27. Barnard, K. R.; Foger, K.; Turney, T. W.; Williams, R. D.; J. Catal. 1990, 125, 265.

28. Lee, Y. Ng.; Lago, R. M.; Fierro, J. L. G. V.; Sapinã, F.; Martínez, E.; Appl. Catal., A 2001, 207, 17.

29. Her, R.; The Chemistry of silica, Wiley: New York, 1979.

30. Dombro, R. A.; Kirch, W.; Eur. Pat. Application 1984, 110, 78; Appl. Catal., A 2001, 207, 17

31. O Connel, M.; Norman, A.; Huttermann, C. F.; Morris, M. A.; Catal. Today 1999, 47, 123

32. Roberts, M. W.; Chem. Soc. Rev. 1989, 18, 451.

33. Tabata, K.; Matsumoto, I.; Kohiki, S.; J. Mater Sci. 1987, 22, 1882.

34. Wu, Y.; Yu, T.; Dou, B. S.; Wang, C. X.; Xie, X. F.; Yu, Z. L.; Fan, S. R.; Wang, L. C.; J. Catal. 1989, 120, 88.

35. Taguchi, H.; Sugita, A.; Nagao, M. J.; Takeda, Y.; J. Solid State Chem. 1995, $116,343$. 